\title{
Applications of natural constraints in critical point theory to boundary value problems on domains with rotation symmetry
}

By

E. W. C. vaN GROESEN*)

1. Introduction. Let $D:=\{r, \theta) \mid 0 \leqq r \leqq 1,-\pi<\theta \leqq \pi\}$ be the unit disc in $\mathbb{R}^{2}$ and let $g:[0,1] \times \mathbb{R} \rightarrow \mathbb{R}$ be a given function. We consider the following nonlinear Dirichlet problem for the Laplacian:

$$
\left\{\begin{array}{ll}
-\Delta u=g(r, u), & (r, \theta) \in D \\
u=0 & \text { for } r=1
\end{array} .\right.
$$

The peculiarity of this BVP is its rotation symmetry: with $u=u(r, \theta)$ a solution of (1.1), for any $\varphi$ the function $R_{\varphi} u:=u(r, \theta+\varphi)$ is also a solution. Calling two functions $u_{1}$ and $u_{2}$ geometrically distinct (as in [4]) if $R_{\varphi} u_{1} \neq u_{2}$ for all $\varphi$, we will derive multiplicity results for geometrically distinct solutions of (1.1). In particular, we shall distinguish between non-radial and radial solutions $u$, depending on whether $u$ depends on the angle variable $\theta$ or not.

Concerning the non-linearity $g$ we require

$$
\begin{array}{ll}
g \in C^{\alpha}([0,1] \times \mathbb{R}, \mathbb{R}) & \text { for some } \alpha \in(0,1], \text { and } u \rightarrow g(\cdot, u) \text { is odd: } \\
g(r, u)=-g(r,-u) & \text { for all } u \in \mathbb{R}, r \in[0,1] .
\end{array}
$$

For various growth conditions on $g$ it is well known that multiplicity results can be obtained using critical point theory for the functional

$$
I(u)=\int_{D} 1 / 2(\nabla u)^{2}-G(r, u), \quad u \in H_{1}^{0}(D)
$$

where

$$
G(r, s)=\int_{0}^{s} g(r, t) \mathrm{d} t
$$

However, in exploiting the $\mathbb{Z}_{2}$ (eveness) invariance of this functional in this specific case, a direct application of (variants of the) Ljusternik-Schnirelmann theory, as in Clark [3], may yield $\mathbb{Z}_{2}$-distinct solutions which are, however, not geometrically distinct. The idea of this paper in deriving existence and multiplicity results for non-radial solutions is to

*) Sponsored by the United States Army under Contract No. DAAG29-80-C-0041. This research has been partially supported by the Niels Stensen Stichting, Amsterdam. 
look for solutions which are odd in $\theta$ and which are periodic with period $2 \pi / k, k \in \mathbb{N}$. That is, for $k \in \mathbb{N}$, we will look for solutions that belong to the set

$$
E_{k}:=\left\{u \in H_{1}^{\circ}(D) \mid u(r, \theta)=-u(r,-\theta) ; u(r, \theta)=u(r, \theta+2 \pi / k)\right\}
$$

Note that any $u \in E_{k}$ has $2 k$ "nodal lines": $u(r, \pi n / k)=0$, for $r \in[0,1]$ and $n \in \mathbb{Z}$, $-k<n \leqq k$. Since $E_{m k} \subset E_{k}$ for any $m \in \mathbb{N}$, we shall say that $u$ has minimal period $2 \pi / k$ if $u \in E_{m k}$ iff $m=1$.

For $k \in \mathbb{N}$, the set $E_{k}$ is a natural constraint for the original critical point problem in the sense (see [6]) that any critical point $u$ of $I$ on $E_{k}$ is a critical point of $I$ on $H_{1}^{0}(D)$ (not conversely, so different from the definitions of Berger [2]) and thus provides a non-radial solution of (1.1) if $u \neq 0$. Moreover, $\mathbb{Z}_{2}$-distinct critical points of $I$ on $E_{k}$ give rise to geometrically distinct solutions of (1.1). The existence and multiplicity of critical points of $I$ on $E_{k}$ is, roughly speaking, determined by the number of those eigenvalues that are "crossed" by the nonlinearity and for which there exists an eigenfunction which belongs to $E_{k}$. This will be shown in section 2: with a sublinear growth condition on $g$, minimization of $I$ on $E_{k}$ will provide solutions of (1.1) with minimal period $2 \pi / k$ and Ljusternik-Schnirelmann theory will give multiplicity results in $E_{k}$ for a restricted set of values $k \in \mathbb{N}$. For superlinear functions $g$, the Mountain Pass theorem (Ambrosetti and Rabinowitz [1]) provides solutions of period $2 \pi / k$ for any $k$ sufficiently large, and if $g$ satisfies an additional monotonicity condition such solutions with minimal period $2 \pi / k$ shall be obtained (generalizing an idea of Nehari [9]) by minimizing $I$ on a natural constraint (of codimension 1) in $E_{k}$.

Radial solutions of (1.1) are found in section 3 by considering $I$ on the set of radial functions. In particular, for the case that $g$ is sublinear we shall modify an other idea of Nehari [10] to construct solutions with a prescibed number of nodes. In this method, the nodes are found in a more or less constructive way.

Concerning non-radial solutions, the only reference we know of is a multiplicity result of Costa and Willem [5] for convex functions $G$ that satisfy stringent growth conditions. For radial solutions, many more results are available: Nehari [10], Hempel [8], Rabinowitz [11], Struwe [12].

Remark. The idea of introducing naturally embedded sets $E_{k}$ as above, originated from the study of periodic solutions in Hamiltonian systems (cf. [6]), and is not restricted to the specific case treated in this paper. In fact, for many problems in which some symmetry is present, such as e.g. for a b.v.p. like (1.1) on a cylindrical domain, square or cube, it is possible to study such "super-harmonic" solutions.

Acknowledgement. A preliminary version of this paper was written while the author was at the M.R.C., Madison, Wi. It is a pleasure to thank Paul Rabinowitz and David Costa for their interest and illuminating discussions.

2. Non-radial solutions. We start with some preliminaries. Denote the succesive eigenvalues of the eigenvalue problem corresponding to (1.1) by $\lambda_{1}<\lambda_{2}<\lambda_{3}<\cdots$. An eigenvalue will be called radial or nonradial depending on whether its eigenfunction is radial 
or not. This makes sense since the radial eigenvalues are simple, with eigenfunction explicitely given by

$$
\mu_{s}^{0}=j_{0, s}^{2}, \quad \psi_{s}^{0}(r)=J_{0}\left(j_{0, s} r\right) \text { for } s \in \mathbb{N},
$$

whereas the non-radial eigenvalues are simple when the eigenfunctions are required to belong to $E_{1}$ : for $k \in \mathbb{N}$

$$
\mu_{s}^{k}=j_{k, s}^{2}, \quad \psi_{s}^{k}(r, \theta)=J_{k}\left(j_{k, s} r\right) \sin k \theta \in E_{k}, \quad \text { for } \quad s \in \mathbb{N} .
$$

Here $J_{k}, k \in \mathbb{N} \cup 0$ are Besselfunctions of the first kind and $j_{k, s}$ denotes the $s$-th strictly positive zero of $J_{k}$.

As stated in the introduction, we will look for critical points of the functional $I$ on the sets $E_{k}, k \in \mathbb{N}$. However, instead of working with the fixed functional $I$ on different sets $E_{k}$, it is somewhat simpler to define different functionals on a fixed set. To that end, let $S$ denote the upper-half of the disc: $S=\{(r, \theta) \mid r \in[0,1], \theta \in[0, \pi]\}$, and write $H=H_{1}^{0}(S)$. For the norm on $H$ we shall take $\|u\|=\left\{\int_{S}|\nabla u|^{2}\right\}^{1 / 2}$. Next, define the following
functionals on $H$ :

$$
\begin{aligned}
& Q_{k}(u):=\int_{0}^{1} \int_{0}^{\pi} 1 / 2\left\{u_{r}^{2}+\frac{k^{2}}{r^{2}} u_{\theta}^{2}\right\} r \mathrm{~d} r \mathrm{~d} \theta, \\
& I_{k}(u)=Q_{k}(u)-\int_{S} G(r, u) .
\end{aligned}
$$

Proposition 2.1. Critical points of the functional $I$ on the set $E_{k}, k \in \mathbb{N}$, are in an one-to-one correspondence with the critical points of the functional $I_{k}$ on $H$.

Proof. This result is an easy consequence of a simple scaling argument, together with standard regularity theory for solutions of elliptic b. v.p.'s. To describe the correspondence, let, for instance, $u \in H$ be a critical point of $I_{k}$. Define its odd continuation to the $\operatorname{disc} D$ :

$$
v(r, \theta):=u(r, \theta) \text { for } \theta \in[0, \pi],-u(r,-\theta) \text { for } \theta \in(-\pi, 0],
$$

and then its $k$-th "subharmonic":

$$
u_{k}(r, \theta):=v(r, k \theta) \text {. }
$$

Then $u_{k} \in E_{k}$ and $u_{k}$ is easily seen to be a critical point of $I$.

Related to proposition 2.1 is the observation that for $k \in \mathbb{N}$ the eigenvalue problem defined by the quadratic functional $Q_{k}$ on $H$ has eigenvalues and eigenfunctions given by

$$
\mu_{s}^{k m}, \psi_{s}^{k, m}(r, \theta):=J_{k m}\left(j_{k m, s} r\right) \sin m \theta, \quad m \in \mathbb{N}, \quad s \in \mathbb{N} .
$$

In particular, since $\psi_{1}^{k, 1}$ is sign definite on $S, \mu_{1}^{k}$ is the smallest eigenvalue and hence

$$
Q_{k}(v) \geqq 1 / 2 \mu_{1}^{k} \int_{S} v^{2} \text { for all } v \in H .
$$


The first result deals with nonlinearities $g$ which are sublinear. Consider the following conditions (expressed in terms of the primitive $G$ defined by (1.3)).

(H1) The function $G$ is subquadratic at infinity in the following sense:

$$
\lim _{|u| \rightarrow \infty} \sup _{\rightarrow} \frac{G(\cdot, u)}{u^{2}}<1 / 2 \lambda_{1}
$$

$(\mathrm{H} 2)_{\gamma}$ There exists a number $\gamma>0$ such that

$$
\liminf _{|u| \rightarrow 0} \frac{G(\cdot, u)}{u^{2}}>1 / 2 \gamma
$$

Theorem 2.2. Let $G$ satisfy $(\mathrm{H} 0),(\mathrm{H} 1)$ and $(\mathrm{H} 2)_{\gamma}$ for some $\gamma>\lambda_{j}, j \in \mathbb{N}$. Then we have for the solutions of BVP (1.1):

(a) If $l$ denotes the number of non-radial eigenvalues not larger than $\lambda_{y}$, then there exist at least 1 non-radial, geometrically distinct solutions.

(b) For each $k \in \mathbb{N}$ for which $\mu_{1}^{k} \leqq \gamma$, there exists at least one solution which has minimal period $2 \pi / k$.

(c) For every pair $(k, s) \in \mathbb{N} \times \mathbb{N}$ for which $\mu_{s}^{k} \leqq \gamma$, there exist at least $s$ distinct solutions with period $2 \pi / k$.

P r o of. Since $0 \leqq Q_{1} \leqq Q_{k}, k \in \mathbb{N}$, and $G$ satisfies (H1), the functionals $I_{k}$ are well defined and weakly lower semi-continuous on $H$. Because of $(H 1)$, these functionals are coercive $\left(I_{k}(u) \geqq I_{1}(u) \rightarrow \infty\right.$ as $\left.\|u\| \rightarrow \infty\right)$ and thus bounded from below on $H$, and satisfy the Palais-Smale condition (see eg. Clark [3]). Since $I_{k}$ is invariant under the action of the group $\mathbb{Z}_{2}=\{i d,-i d\}$, we shall apply Ljusternik-Schnirelmann theory as in [3] and use the genus of symmetric, compact subsets of $H \backslash\{0\}$ as index theory. This theory provides the existence of at least $m \mathbb{Z}_{2}$-distinct critical points of $I_{k}$ if some set $\Sigma$ of genus $m$ can be found such that $I_{k}(\Sigma)<0$. We shall specify below the sets $\Sigma$ which will yield the desired multiplicity results. To prove (a), let $k=1$ and denote by $v_{m}, 1 \leqq m \leqq l$, those non-radial eigenfunctions $\psi_{s}^{n}, n \in \mathbb{N}, s \in \mathbb{N}$ which correspond to eigenvalues not larger than $\lambda_{j}$. Consider for $\varrho>0$ the set $\Sigma^{\varrho}=\left\{v=\sum_{1}^{l} \alpha_{m} v_{m} \mid \alpha_{m} \in \mathbb{R}, 1 \leqq m \leqq l,\|v\|=\varrho\right\}$. Then, by definition of $v_{m}, Q_{1}(v) \leqq \lambda_{j} \int v^{2}$ for every $v \in \Sigma^{o}$. Furthermore, since $G$ satisfies $(\mathbf{H} 2)_{\gamma}$, for every $v \in \Sigma^{\varrho}$ it holds $\int G(\cdot, v)>1 / 2 \gamma \int v^{2}$ for $\varrho$ sufficiently small. Since $\Sigma^{\varrho}$ is a compact set, and $\gamma \geqq \lambda_{j}$, it follows that there exists a $\varrho>0$ sufficiently small such that $I_{1}(v)<0$ for every $v \in \Sigma^{\varrho}$. Since $\Sigma^{e}$ has genus $l$, part (a) follows with proposition 2.1 .

To prove part (b), we minimize $I_{k}$ on $H$. If $u$ is such a minimizer, and $\mu_{1}^{k} \leqq \gamma$, then $I_{k}(u)$ is negative since $I_{k}\left(\varrho \psi_{1}^{k}\right)=1 / 2 \mu_{1}^{k} \varrho^{2} \int\left\{\psi_{1}^{k}\right\}^{2}-\int G\left(\varrho \psi_{1}^{k}\right)<1 / 2 \varrho^{2}\left(\mu_{1}^{k}-\gamma\right) \int\left\{\psi_{1}^{k}\right\}^{2}$ is negative for $\varrho$ sufficiently small. Moreover, such a minimizer is sign definite on $H$ : if $u$ were not sign definite, either its positive part $u_{+}(\cdot):=\max \{u(\cdot), 0\}$ or its negative part $u_{-}(\cdot)=\min \{u(\cdot), 0\}$ would yield a smaller value for $I_{k}$. Then, via proposition 2.1 , this function corresponds to a solution of (1.1) that belongs to $E_{k}$ but (being sign definite for $\theta \in(0, \pi / k))$ not to $E_{k \cdot m}$ for $m \geqq 2$ and thus has minimal period $2 \pi / k$. 
For $k \in \mathbb{N}$ consider for $\varrho>0$ the set

$$
\Sigma^{\varrho}:=\left\{v=\sum_{t=1}^{s} \alpha_{t} \psi_{t}^{k, 1} \mid \alpha_{t} \in \mathbb{R}, 1 \leqq t \leqq s:\|v\|=\varrho\right\} .
$$

Then, as above, now using (2.6), it can be verified that $I_{k}\left(\Sigma^{\varrho}\right)<0$ if $\mu_{s}^{k} \leqq \gamma$ and $\varrho>0$ is sufficiently small. Since $\Sigma^{\varrho}$ has genus $s$, part (c) follows with proposition 2.1 . This completes the proof.

The next results deal with nonlinearities which are superlinear. Consider the following conditions.

(H3) There exist numbers $\mu>2$ and $R>0$ such that

$$
g(\cdot, u) \cdot u \geqq \mu G(\cdot, u)>0 \quad \text { for all } u \text { with }|u|>R,
$$

and numbers $M_{1,2}>0$ and $r>2$ such that

(H4) The function $u \rightarrow g(\cdot, u)$ is differentiable, derivative $g^{\prime}$, and the following conditions are satisfied:

(i) $G(\cdot, u)>0$ for all $u \neq 0$,

(ii) $g^{\prime}(\cdot, u) u^{2}-g(\cdot, u) \cdot u>0$ for all $u \neq 0$.

The well known inequality (2.7) can be integrated to give

$$
G(\cdot, u) \geqq a+b|u|^{\mu} \text {. for all } u \in \mathbb{R}
$$

for some constants $a$ and $b>0$. Condition (2.8), polynomial growth at infinity, implies by Sobolev embedding theorems for functions on the plane that the functional $\int G(\cdot, u)$ is well defined and weakly continuous on $H_{1}^{0}$. (H4) is essentially a monotonicity condition on the growth of $g$; any positive, homogeneous function $G$ of degree larger than 2 , and finite sums of such functions, satisfy this condition.

Theorem 2.3. Let $G$ satisfy (H0) with $\alpha=1$ and (H3). There exist $k_{0} \in \mathbb{N}$ and for each $k \in \mathbb{N}, k \geqq k_{0}$, a solution $u_{k}$ which has period $2 \pi / k$. Consequently, there exist infinitely many, geometrically distinct, non-radial solutions.

P r o of. We use the mountain pass lemma (Ambrosetti \& Rabinowitz [1]) to show that $I_{k}$ has a critical point on $H$ for $k$ sufficiently large, from which the result follows with proposition 2.1 .

For $k \in \mathbb{N}, I_{k}$ is well defined on $H$ and as a consequence of (2.7), (2.9) satisfies the Palais-Smale condition (see [1]) and $I_{k}(\varrho v) \rightarrow-\infty$ as $\varrho \rightarrow \infty$ for every $v \in H$. To apply the mountain pass lemma it only remains to show that there exist numbers $\varrho>0$ and $\alpha>0$ such that

$$
I_{k}(v) \geqq \alpha>0 \quad \text { for every } \quad v \in H,\|v\|=\varrho .
$$

To that end, note that since $g$ is locally Lipschitz, there exist constants $M>0$ and $\delta>0$ such that $G(\cdot, u) \leqq M u^{2}$ for all $u \in \mathbb{R},|u| \leqq \delta$, and together with (2.9) it follows that for 
some $M_{0}>0: G(\cdot, u) \leqq M u^{2}+M_{0}|u|^{r}$ for all $u \in \mathbb{R}$. Then, by Sobelev's embedding theorem, (2.6) and the inequality $Q_{k}(v) \geqq 1 / 2\|v\|^{2}$ on $H$, it follows that

$$
I_{k}(u) \geqq 1 / 2\|u\|^{2}-M \int u^{2}-M_{0} \int|u|^{r} \geqq\left(1 / 2-M / \mu_{1}^{k}\right)\|u\|^{2}-c \cdot M_{0}\|u\|^{r} .
$$

Since $\mu_{1}^{k} \rightarrow \infty$ as $k \rightarrow \infty$, it follows that $I_{k}$ satisfies (2.10) for some $\varrho>0$ and $\alpha>0$ provided $k \geqq k_{0}$, where $k_{0}$ is such that $\mu_{1}^{k_{0}}>2 M$. This proves $(2.10)$.

The proof of the next result provides a much more explicite characterization of the functions $u_{k}$ from theorem 2.3.

Theorem 2.4. Let $\mathrm{G}$ satisfy (H0), (H3) and (H4). Let $k_{0} \in \mathbb{N}$ be such that $g^{\prime}(\cdot, 0)<\mu_{1}^{k_{0}}$. Then, for any $k \in \mathbb{N}, k \geqq k_{0}$, there exists a function $u_{k}$ which has minimal period $2 \pi / k$.

P r o of. For any $k \geqq k_{0}$ we shall prove the existence of a sign definite critical point of $I_{k}$ on $H$, from which the result follows with proposition 2.1. Using the same ideas as in [6], [7], consider for $k \geqq k_{0}$ the following analytical mini-max problem ("analytical" to distinguish from the topological mini-max formulation in the proof of the mountain pass lemma)

$$
\inf _{v \in H,\|v\|=1} \sup _{\varrho>0} I_{k}(\varrho v) .
$$

For fixed $v \in H,\|v\|=1$, the (twice differentiable) function $0 \leqq \varrho \rightarrow I_{k}(\varrho v)$ vanishes at $\varrho=0$, is positive for $\varrho>0$ small (since $k \geqq k_{0}$ ) and tends to $-\infty$ as $\varrho \rightarrow \infty$. An investigation of the second derivative of this function at its critical point(s), shows that condition (H4) (ii) implies that this function has, in fact, a unique critical point, say $\varrho=\varrho(v)$, viz. the point at which $I_{k}(\varrho(v) v)$ is maximal. The set of points $N_{k}:=\{\varrho(v) v \in H \mid\|v\|=1\}$ can therefore also be described as

$$
N_{k}=\left\{u \in H \backslash\{0\} \mid\left\langle I_{k}^{\prime}(u), u\right\rangle=0\right\},
$$

where $\left\langle I_{k}^{\prime}(u), \cdot\right\rangle \in H^{*}$ denotes the first derivative of $I_{k}$ at $u$. (This set $N_{k}$ has been considered for the first time, for a specific case, by Nehari [10].) It is not difficult to show (see also [7]) that $N_{k}$ is a smooth manifold (in particular, $u=0$ is an isolated point of $\left\langle I_{k}^{\prime}(u), u\right\rangle=0$ since $\left.k \geqq k_{0}\right)$ with codimension 1 , and that $N_{k}$ is a natural constraint for $I_{k}$ : any critical point of $I_{k}$ on $N_{k}$ is also a critical point of $I_{k}$ on $H$ (the Lagrange multiplier vanishes). The analytical mini-max problem (2.11) is thus equivalent with the minimization problem for $I_{k}$ on $N_{k}$ :

$$
\inf \left\{I_{k}(u) \mid u \in N_{k}\right\} \text {. }
$$

It remains to show that (2.13) has a sign definite solution. Since the set $N_{k}$ is not weakly closed in $H$, this is not completely standard and we will indicate the main ideas (see also [7]).

Denote the value of (2.13) by $c_{k}$; then $c_{k}$ is strictly positive. Next, note that for $u \in N_{k}$, $I_{k}(u)=\int W(u)$, where the function $W(u)=1 / 2 g(\cdot, u) u-G(\cdot, u)$ satisfies for any $u \in \mathbb{R}$, $W(\varrho u)>W(u)$ for every $\varrho>1$ (as a consequence of (H.4) (ii)). Now, consider a minimizing sequence $\left(u_{m}\right), m \in \mathbb{N}$. Since $u_{m} \in N_{k}$, it readily follows from (2.7) that $\left(u_{m}\right)$ is uniformly 
bounded in $H$ and, hence, has a weakly convergent subsequence, $u_{m} \rightarrow \hat{u}$ say. Since the functionals $\int W(u)$ and $\int g(\cdot, u) u$ are weakly continuous, it follows that $\int W(\hat{u})=c_{k}$ and $\left\langle I_{k}^{\prime}(\hat{u}), \hat{u}\right\rangle \leqq 0$. To show that $\hat{u}$ belongs, in fact, to $N_{k}$ and is thus a minimizer for (2.12), first note that $\hat{u}=0$ is excluded since $u_{m} \in N_{k}$ and $u_{m} \rightarrow 0$ would imply that $\left\|u_{m}\right\| \rightarrow 0$, contradicting the fact that 0 is an isolated point of $N_{k} \cup\{0\}$. Hence $\hat{u} \neq 0$. Now, if $\left\langle I_{k}^{\prime}(\hat{u})\right.$, $\hat{u}\rangle$ were negative, there would exist a $\varrho<1$ such that $\varrho \hat{u} \in N_{k}$ (definition of $N_{k}$ ). But then $\int W(\varrho \hat{u})<\int W(\hat{u})=c_{k}$, contradicting the definition of $c_{k}$. This proves the existence of a minimizer for (2.13). Since $N_{k}$ is a symmetric set, it is standard to show that such a minimizer is sign definite on $S$, which completes the proof.

3. Radial solutions. In this section we shall briefly investigate radial solutions of (1.1) by looking for critical points of $I$ on the set of radial functions:

$$
R:=\left\{u \in H_{1}^{0}(D) \mid u(r, \theta)=u(r)\right\} .
$$

If $g$ is sublinear, i.e. satisfies ( $\mathrm{H} 0)$, (H1) and ( $\mathrm{H} 2)_{\gamma}$ for some $\gamma \geqq \lambda_{j}, j \in \mathbb{N}$, the functional $I$ is bounded from below on $R$ and standard Ljusternik-Schnirelmann theory (as in [3]) shows that there exist $k$ distinct radial solutions, where $k$ is the number of radial eigenvalues not larger than $\lambda_{j}$. If $g$ is superlinear, i.e. satisfies (H0) and (H3), the existence of infinitely many radial solutions is well established (see e.g. [9, 10,11, 12]). In particular, Nehari's idea ([10]) to introduce a natural constraint for problems with a nonlinearity that belongs to a restricted class of functions, can be modified to be applicable to any function $g$ which satisfies (H4), and provides solutions with a prescribed number of nodes. Nodal properties of solutions are also present in the references cited above, but Nehari's idea seems particularly useful because it characterizes the nodes in a more or less explicit way. In this section we shall modify this idea to the case of sublinear nonlinearities $g$. Then, in contrast to the superlinear case, the behaviour of $g$ at the origin determines a lower bound for the length of the intervals on which a nontrivial solution can exist.

Theorem 3.1. Let $G$ satisfy $(\mathrm{H} 0),(\mathrm{H} 1)$ and $(\mathrm{H} 2)_{\gamma}$ with $\gamma \geqq \mu_{j+1}^{0}$ for some $j \in \mathbb{N}$. Suppose, moreover, that $g(r, u)=0$ iff $u=0$ and that $g(\cdot, u)$ is strictly concave for $u>0$. Then, for each $k \in \mathbb{N} \cup\{0\}, 0 \leqq k \leqq j$, there exists at least one pair of solutions which have precisely $k$ nodes in the interval $(0,1)$.

Proof. It is a standard result that the weakly lower semi-continuous, coercive functional $I$ is bounded from below on $R$ and attains its (negative, since $\gamma>\mu_{1}^{0}$ ) minimum value for at least one non-trivial function $u \in R$. Due to this extremal characterization, the solution is easily seen to be sign definite on $(0,1)$. The strict concavity of $g$ for $u>0$ implies that sign definite solutions of (1.1) are unique up to sign, which proves the existence of precisely one solution (up to sign) without interior nodes. The essential idea of the rest of the proof is best explained for the simplest case $j=1$, so we shall restrict ourselves to that case. Define for $\alpha \in[0,1]$ the sets of functions which are identically zero on the left or on the right of $\alpha$ :

$$
\begin{array}{lll}
R_{1}(\alpha):=\{u \in R \mid u(x)=0 & \text { for } & x \in[\alpha, 1]\} \\
R_{2}(\alpha):=\{u \in R \mid u(x)=0 & \text { for } & x \in[0, \alpha]\}
\end{array}
$$


Then consider the functions $m_{i}, i=1,2$ and $f$ defined on $[0,1]$ by:

$$
\begin{aligned}
m_{i}(\alpha): & =\inf \left\{I(u) \mid u \in R_{i}(\alpha)\right\}, \\
f(\alpha) & =m_{1}(\alpha)+m_{2}(\alpha) .
\end{aligned}
$$

Note that $m_{1}$ and $m_{2}$ are finite, non-positive functions with $m_{1}(0)=0=m_{2}$ (1). In fact, the infima in the definitions of $m_{i}$ are attained by functions $w_{i}(\cdot, \alpha)$, say, which are unique up to sign. Furthermore, there exist numbers $\alpha_{1}>0$ and $\alpha_{2}<1$ such that $w_{1}(\cdot, \alpha) \equiv 0$ for $\alpha \in\left[0, \alpha_{1}\right]$ and $w_{2}(\cdot, \alpha) \equiv 0$ for $\alpha \in\left[\alpha_{2}, 1\right]$. The functions $m_{i}$ are differentiable with derivatives:

$$
\begin{aligned}
& m_{1}^{\prime}(\alpha)=-1 / 2 \alpha\left\{\partial_{r} w_{1}(\alpha-0, \alpha)\right\}^{2}, \\
& m_{2}^{\prime}(\alpha)=1 / 2 \alpha\left\{\partial_{r} w_{2}(\alpha+0, \alpha)\right\}^{2} .
\end{aligned}
$$

The numbers $\alpha_{1}$ and $\alpha_{2}$ depend on the behaviour of the function $g$ at the origin. As a consequence of the assumption $\gamma \geqq \mu_{2}^{0}$ (for $j=1$ ) it follows that $\alpha_{1}<\alpha_{2}$. Indeed, if $\alpha^{*} \in(0,1)$ denotes the node of the eigenfunction corresponding to $\mu_{2}^{0}$, then $\alpha_{1}<\alpha^{*}<\alpha_{2}$. For instance, to show $\alpha_{1}<\alpha^{*}$ observe that $\mu_{2}^{0}$ is the lowest eigenvalue of the eigenvalue problem on $\left[0, \alpha^{*}\right]$ and, since $\gamma \geqq \mu_{2}^{0}, m_{1}\left(\alpha^{*}\right)$ is negative. In the same way $\alpha^{*}<\alpha_{2}$. From these observations it follows that the function $f$ is strictly negative, differentiable and that it attains its maximum at some interior point $\hat{\alpha} \in(0,1)$. Then, the function $\hat{u}$ defined by

$$
\hat{u}(\cdot)=\left|w_{1}(\cdot, \hat{\alpha})\right|-\left|w_{2}(\cdot, \hat{\alpha})\right|
$$

belongs to $R$, is differentiable on $[0,1]$ (in particular at $x=\hat{\alpha}$, since $\left.f^{\prime}(\hat{\alpha})=0\right)$ and, hence, is a smooth solution of (1.1) which has precisely one interior node, namely $\hat{\alpha}$. This completes the proof of the theorem for $j=1$. The same idea generalizes to arbitrary $j \in \mathbb{N}$.

\section{References}

[1] A. Ambrosetti and P. H. Rabinowitz, Dual variational methods in critical point theory and applications. Funct. Anal. 14, 349-381 (1973).

[2] M. S. Berger, Nonlinearity and Functional Analysis. New York 1977.

[3] D. C. Clark, A variant of Ljusternik-Schnirelmann theory. Indiana Univ. Math. J. 22, 65-74 (1972).

[4] D. G. Costa, An application of the Ljusternik-Schnirelmann theory. Proceedings of 15th Brazilian Seminar of Analysis, 211-223 (1982).

[5] D. G. Costa and M. WILlem, Multiple critical points of invariant functionals and applications. MRC Report 2532, June 1983.

[6] E. W. C. VAN GroESEN, Applications of natural constraints in critical point theory to periodic solutions of natural Hamiltonian systems. MRC Report 2593, November 1983.

[7] E. W. C. VAN GROESEN, On small period, large amplitude normal modes of natural Hamiltonian systems. MRC Report 2637; January 1984.

[8] J. A. HeMPEL, Multiple solutions for a class of nonlinear boundary value problems. Indiana Univ. Math. J. 20, 983-996 (1971).

[9] Z. NeHARI, On a class of nonlinear second order differential equations. Trans. Amer. Math. Soc. 95, $101-123(1960)$. 
[10] Z. NEHARI, Characteristic values associated with a class of nonlinear second-order differential equations. Acta Math. 105, 141-175 (1961).

[11] P. H. RABINOWITZ, A note on a nonlinear eigenvalue problem for a class of differential equations. J. Differential Equations 9, 536-548 (1971).

[12] M. STRUWE, Infinitely many solutions of superlinear boundary value problems with rotational symmetry. Arch. Math. 36, 360-369 (1981).

Eingegangen am 5.1.1984*)

Anschrift des Autors:

E. W. C. van Groesen

Mathematical Institute

Catholic University of Nijmegen

Toernooiveld

6525 ED Nijmegen

The Netherlands

*) Eine Neufassung ging am 20.7. 1984 ein. 